\title{
SIGNIFICANCE OF EXPERT COMPETENCE CONSIDERATION WHILE GROUP DECISION-MAKING USING AHP
}

\author{
V.V.Tsyganok* \\ E-mail: vitaliy.tsyganok@gmail.com \\ S.V.Kadenko \\ E-mail: $\underline{\text { sergeykadenko@mail.ru }}$ \\ O.V.Andriichuk \\ E-mail: andreychuck@ukr.net \\ National Academy of Sciences of Ukraine \\ The Institute for Information Recording \\ Kyiv, Ukraine
}

\begin{abstract}
The question addressed in the paper is: "Is it always necessary to take expert competence coefficients into consideration while aggregating individual expert judgements us ing AHP?" Some recent studies show that while aggregating direct cardinal and ordinal individual estimates expert competence does not matter significantly in cases when the number of expert group members is relatively large (more than 50 experts). The present study is aimed at clarifying the issue in case individual expert judgements are provided as pair comparisons in fundamental scale and aggregated using AHP methodology. Expert estimates are modelled based on presumptions that they are distributed according to exponential and absolute normal distribution laws. Experiment results confirm the presumption that under large expert numbers competence of individual experts does not matter significantly and can be neglected, but if the number of experts in a group is relatively small, expert competence should always be taken into consideration.
\end{abstract}

Keywords: AHP/ANP, expert competence, group decision-making, judgement, estimate

\section{Introduction}

Taking expert competence into account while expertise conduction is a rather laborious procedure, since it involves estimating expert competence evaluation in one way or another, so, obviously, in order to reduce expertise cost we should try to exclude this stage while group decision-making support (certainly not at the price of expertise result adequacy). So, let us focus on necessary conditions of expert competence consideration while individual expert estimate aggregation.

There is a presumption, that the significance of expert competence consideration while group decision-making is inversely proportional to expert group size. So, consequently, after exceeding a certain expert group size, it is inappropriate to take expert competence into account. The hypothesis is confirmed by research conducted while direct estimation of a certain single object (Zagoruiko, 1988; Liubchenko, 2005) and, as a result, it was concluded that under maximal acceptable expert estimation error of $20 \%$ and expert group size of at least 30 experts, the differences in expert competence can be neglected.

We conducted similar research for the case of group ordinal expert estimation. As for the case of expert competence consideration significance while building pair comparisons in the AHP (Saaty,

\footnotetext{
* Corresponding author
} 
1980), we are not aware of any research having been made in this direction. So, our paper addresses this specific issue.

We tend to believe that expert competence consideration significance while using AHP during group expert examination depends on individual expert estimate (preference) distribution law and on the number of alternatives estimated.

\section{Modelling the experiment}

\subsection{Expert estimation process simulation}

Let us imitate expert estimation process (i.e. results of pair comparisons in the Fundamental scale) using the Monte-Carlo method, since involving real experts is a costly procedure problematic to organize. Let us follow the non-parametric approach to expert estimation modelling, suggested by (Orlov, 1996), since it is virtually hopeless to try to determine the expert estimate distribution law in the ge neral case. In our opinion, the expert estimate distribution law depends on multiple features of a specific expertise and a specific expert (his knowledge in a given domain, his understanding of the expertise subject, the scale he estimates the alternatives in etc), so it is inappropriate to try searching for a universal distribution law for any type of expert estimates. Thus, let us consider the experimental function behaviour based on several possible expert estimate distributions.

Even distribution is unsuitable, because when the number of modelled expert estimates is fairly large, multiple "opposite" opinions appear, and aggregate alternative estimates are too unstable. So we consider the expert group competent enough, so that the experts' estimates lie close to certain true values (representing the mathematical expectation of modelled random estimates). Let us consider requirements to resulting distribution of modelled experts' estimates:

1) probability density function (PDF) must be defined on all Fundamental scale range;

2) the function must be continuous on all its definitional domain;

3) the function should have a maximum in some given „true" value;

4) distance from a given true value to points with equal values of function should satisfy the ratio scale requirements.

According to the above-listed items we propose to simulate individual expert estimates specifying deviation from a given true value. The deviation shows how many times in one direction or another, the true value is changed. In other words, the deviation is a random value which multiplies or divides the given true value to simulate the expert estimate.

\subsection{Modelling de viation}

Let us specify deviation as a random value distributed according to a certain law in the $[1, \infty)$ range, where the only $\operatorname{PDF} f(x)$ maximum is achieved in the point $x=1$. After that, a mathematical operation on the deviation (multiplication or division) is chosen uniformly. Thus, we can formulate requirements to deviation value distribution law:

1) PDF must be defined in the range $[1 ; \infty)$, but the interval, where significant part of the function lies and where random value belongs with confidence probability of 0.95 , should be the interval $[1 ; 2]$ (it means the experts' probable error does not exceed 100\%);

2) the function must be continuous in all its definitional domain;

3 ) in point $x=1$ there is a unique (the only) maximum of the function (the function is a monotonically decreasing one).

\subsection{Suggested expert estimate distribution laws}

According to the requirements, we suggest studying the cases when individual expert estimates' deviation from a given true value is distributed according to exponential and half-normal (absolutenormal, special case of folded normal) laws (see Fig. 1). 


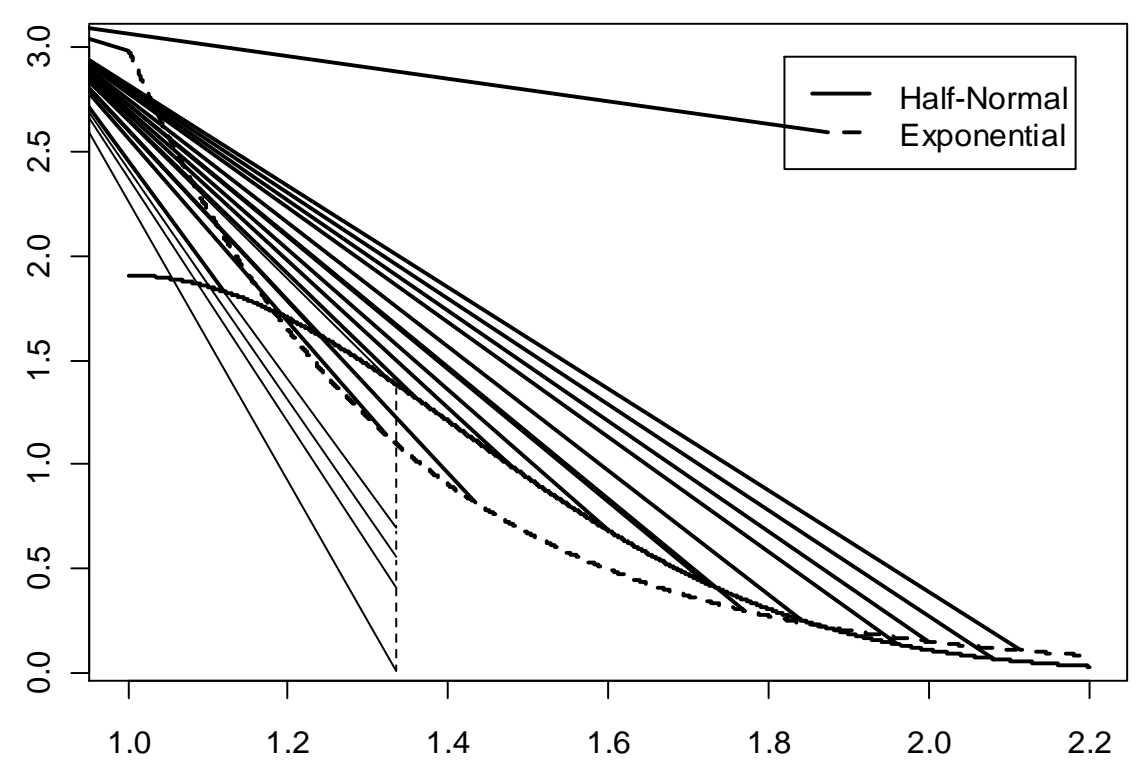

Fig. 1 Distribution laws of individual expert estimates' deviation from the given "true" value.

PDFs, shown on Fig. 1 are shifted by 1 to the right in order to allow using their random values as multipliers (whose values in this case would lie close to $x=1$ ).

The PDF of initial exponential distribution is:

$f(x)=\lambda \cdot e^{-\lambda x}, x \geq 0$,

where $\lambda>0$ is the parameter of the distribution, often called the rate parameter.

The half-normal distribution is derived from the normal distribution, having the PDF (known as the Gaussian function):

$f(x)=\frac{1}{\sqrt{2 \pi \sigma^{2}}} e^{-\frac{(x-\mu)^{2}}{2 \sigma^{2}}}, x \in(-\infty ; \infty)$,

where parameter $\mu$ is the mean / expected value (location of the peak) and $\sigma^{2}$ is the variance (the measure of the width of the distribution).

So the PDF for initial (non-shifted) half-normal distribution (at $\mu=0$ ) is shown as follows:

$f(x)=\frac{2}{\sqrt{2 \pi \sigma^{2}}} e^{-\frac{x^{2}}{2 \sigma^{2}}}, x \in[0 ; \infty)$.

In addition, functions' parameters $\sigma$ and $\lambda$ are fitted so that distributions' mathematical expectations are equal. So mathematical expectation for exponential distribution law equals $1 / \lambda$, and for halfnormal law it's $\sqrt{2 / \pi} \cdot \sigma$. In order for the deviation value distribution law to fulfil the first aforementioned requirement (the experts' probable error is not allowed to exceed 100\%) we can fit appropriate mathematical expectation for both distributions: from cumulative distribution function for exponential distribution $1-e^{-\lambda x}=0.95 \Rightarrow 1 / \lambda=-x / \ln (0.05)$ (in our case $x=1$ ) and it equals approximately $0.3338(33.38 \%)$. So we can calculate parameters for both distributions based on following relation: $1 / \lambda=\sqrt{2 / \pi} \cdot \sigma=0.3338$.

\subsection{Setting "true" al ternative weight values and pair comparison matrices}

Modelled expert estimates, lying within the vicinity of the true value, are given in the Fundamental scale. So, let us define the true alternative weight values $w_{i}, i=\overline{1, m}$ (they should lie within the same order of magnitude and be distinguishable (Saaty, 2008)). Without loss of generality, and for convenience's sake, let us presume that given alternative weights are sorted in order of decrement. Now let us assign a weight (for example, $w_{1}=10$ ) to the $1^{\text {st }}$ alternative and define other weights based on pre- 
sumption that in order for alternatives to be distinguishable, there should be at least a 5 per cent difference between their weights, and, on the other hand, they should belong to the same order of magnitude. Such an array of weights can be built according to the following recursive formula: $w_{i+1}=w_{i} / \operatorname{random}\left[1.05 ;(m-i) \sqrt[10 \prod_{k=1}^{i-1} \frac{w_{k+1}}{w_{k}}]{ }\right]$, where random - is a function, generating a random value from a given range.

For example, based on the recursive formula we've obtained non-normalised weights for various numbers of alternatives $m$ (see table 1).

Table 1. True alternative weights, generated for various numbers of alternatives.

\begin{tabular}{|c|c|c|c|c|c|c|c|c|c|}
\hline $\begin{array}{c}m \text { (number of } \\
\text { alternatives) }\end{array}$ & $w_{1}$ & $w_{2}$ & $w_{3}$ & $w_{4}$ & $w_{5}$ & $w_{6}$ & $w_{7}$ & $w_{8}$ & $w_{9}$ \\
\hline 3 & 10 & 7.5826 & 1.2476 & & & & & & \\
\hline 4 & 10 & 5.3495 & 4.7273 & 1.5106 & & & & & \\
\hline 5 & 10 & 8.9470 & 7.7068 & 5.4742 & 1.3742 & & & & \\
\hline 6 & 10 & 7.6739 & 6.5496 & 4.4959 & 3.0977 & 1.1034 & & & \\
\hline 7 & 10 & 7.3787 & 6.7863 & 5.5722 & 4.4722 & 3.5430 & 1.1577 & & \\
\hline 8 & 10 & 7.2015 & 5.4130 & 4.0386 & 3.4689 & 2.6224 & 1.6240 & 1.0927 & \\
\hline 9 & 10 & 8.4819 & 6.9407 & 6.3836 & 5.0463 & 3.4231 & 2.3741 & 2.0969 & 1.1204 \\
\hline
\end{tabular}

Then, based on this sequence of generated true alternative weights, we can form an ideally consistent pair comparison matrix (PCM) using the equation: $a_{i j}=w_{i} / w_{j}$. Hereafter we consider the PCM reciprocally symmetrical: $a_{i j}=1 / a_{j i}$, and so use only the elements lying above its principal diagonal ( $i<j$ ). Since true alternative weights are sorted in the order of decrement and all alternatives are distinguishable, all elements above the principal diagonal will fulfil the following condition: $a_{i j}>1 \mid i<j$. Besides, while defining true weights' interrelations, we consider the Fundamental scale as continuous, and so, consequently, the elements of the ideally consistent PCM will represent real and not only integer values $\left(a_{i j} \in \mathfrak{R}\right)$.

\subsection{Fluctuating the PCM}

Now let us define a certain random fluctuation of the PCM formed above. Moreover, these random deviations of PCM elements' deviations from true values (let us denote them as $\Delta$ ) should be given in ratio scale, i.e. the follow ing equation must be fulfilled: $\Delta=\left(a_{\Delta^{+}} / a_{\mathrm{e}}\right)=\left(a_{\mathrm{e}} / a_{\Delta_{-}}\right)$, where $a_{\mathrm{e}}$ is the value of ideally consistent PCM element, subject to fluctuation, $a_{\Delta^{+}}$is the value of this same element in case of its increment and $a_{\Delta-}$ is the value in case of its decrement. The random value of $\Delta$ is defined according to one of the aforementioned distribution laws (exponential or half-normal). Since the probability distribution functions of these laws are defined in the positive range (to the right from zero point), and the deviation is multiplicative, let us increase the generated random value by $1(\Delta=\Delta+1)$. The deviation direction (positive $\left(a_{\Delta^{+}}=a_{\mathrm{e}} \Delta\right)$ or negative $\left.\left(a_{\Delta-}=a_{\mathrm{e}} / \Delta\right)\right)$ is chosen randomly, so, based on aforementioned distributions, defined on positive semi-axis (to the right from zero point), we get symmetrical distribution laws. And, since the elements of modelled individual expert PCM should belong to the discrete Fundamental scale, we shall approximate real values obtained in the described way with the nearest Fundamental scale mark. Thus, the elements of individual PCM can assume the values of: $\{1 / 9,1 / 8, \ldots, 1 / 2,1,2, \ldots, 8,9\}$.

\subsection{Estimate aggregation rules}

The next experiment step is the aggregation of just mode lled PCM. We suggest aggregating the PCM using the geometric mean of respective matrix elements. We suggest calculating two variants of the aggregate matrix: with different individual expert competence values and with equal ones. The $i$-th 
expert competence $c_{i}$ is modelled as evenly distributed random value. In this case the aggregate matrix elements are calculated according to equations:

$a_{i j}^{\prime}=\sqrt[n]{\prod_{k=1}^{n} a_{i j k}}-$ without competence consideration;

$a_{i j}^{\prime \prime}=\left(\sum_{x=1}^{n} c_{x}\right) \sqrt{\prod_{k=1}^{n} a_{i j k}^{c_{k}}}-$ with competence consideration.

Based on these two aggregate matrices alternative weights are calculated using the eigenvector method $w_{i}^{\prime}$ and $w_{i}^{\prime \prime}, i=\overline{1, m}$. The relation value $\xi=\max _{i}\left(\max \left(w_{i}^{\prime}, w_{i}^{\prime \prime}\right) / \min \left(w_{i}^{\prime}, w_{i}^{\prime \prime}\right)\right)$ represents the difference of expert estimation results with and without expert competence consideration.

\subsection{Outline of the results}

The experiment was conducted for different expert numbers $n$, where $n \in[3 ; 200]$ and different quantities of estimated alternatives $m$ where $m \in[3 ; 9]$ ( $m$ is defined based on human psychophysiological constraints, allowing the expert to analyse no more than $7 \pm 2$ object simultaneously (Miller, 1956)). One more experiment parameter is the distribution law of individual expert estimates (two variants under consideration are mentioned above).

To ensure statistical reliability of modelling results for fixed confidence interval $\beta$ it is necessary to perform no less than $N$ repetitions of experiment: $N \geq D_{\xi}\left(P_{\beta} / \beta\right)$, where $D_{\xi}$ is the dispersion of the random value under consideration; $P_{\beta}$ is the expectancy of getting the random value lying within the specified interval $\beta$. Thus, to ensure getting of a random value in the confidence interval $\beta= \pm 5 \%$ with expectancy $P_{\beta}=95 \%$ and under $D_{\xi} \approx 10 \%$, the experiment is repeated for $N=200$ times.

To realise modelling experiment special software was developed (see screenshot on Fig.2).

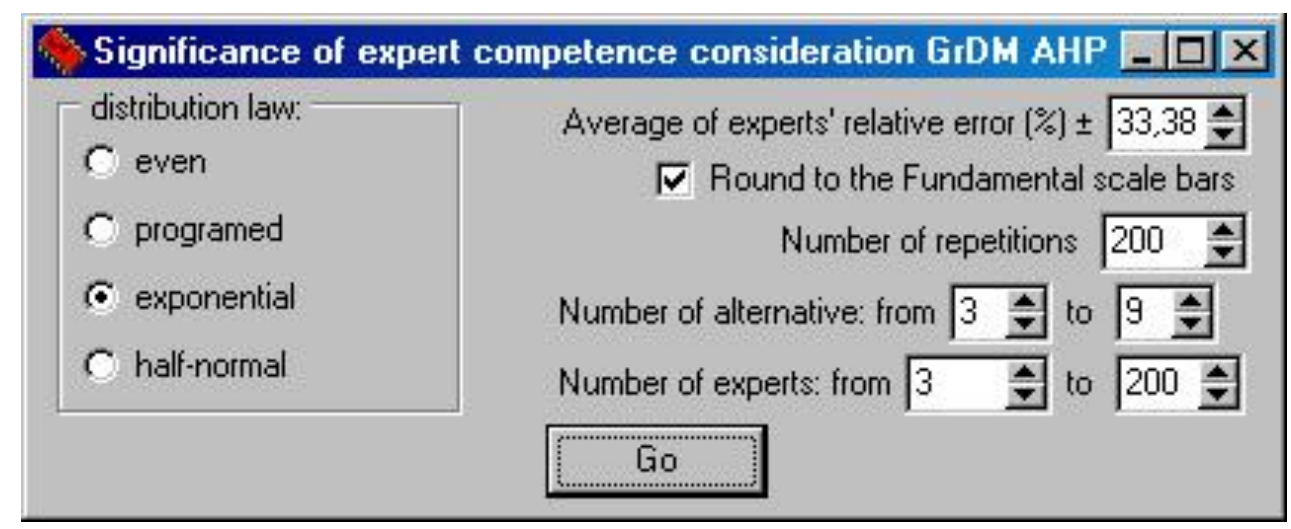

Fig.2. Screenshot of modelling software's input interface.

The software's interface allows to choose series of model parameters: distribution law of the deviation value (even, exponential, half-normal), mathematical expectations of the distribution law (average of experts' relative error), the number of experiment's repetitions $(N)$, variable number of alternatives $(m \in[3 ; 9])$ and experts $(n \in[3 ; 200])$, as well as define if elements of modelled individual expert PCM should rounded to the nearest Fundamental scale marks or not.

As a result, two variants of dependence between $\xi$ and the number of experts in a group under different expert group sizes $(n)$ and different number of alternatives $(m)$ are obtained (Fig. $3 \mathrm{a}-$ for exponential expert estimate deviation distribution law, Fig. $3 \mathrm{~b}$ - for half-normal law). As we can see from the Fig.3, the plots go down below significance level (5\% of differences between aggregate expert estimates obtained for equal and different expert competence cases) when the number of experts in a 
group equals about 50 for both deviation distribution laws. It means that under these and larger quantities of experts in a group expert competence may be neglected.

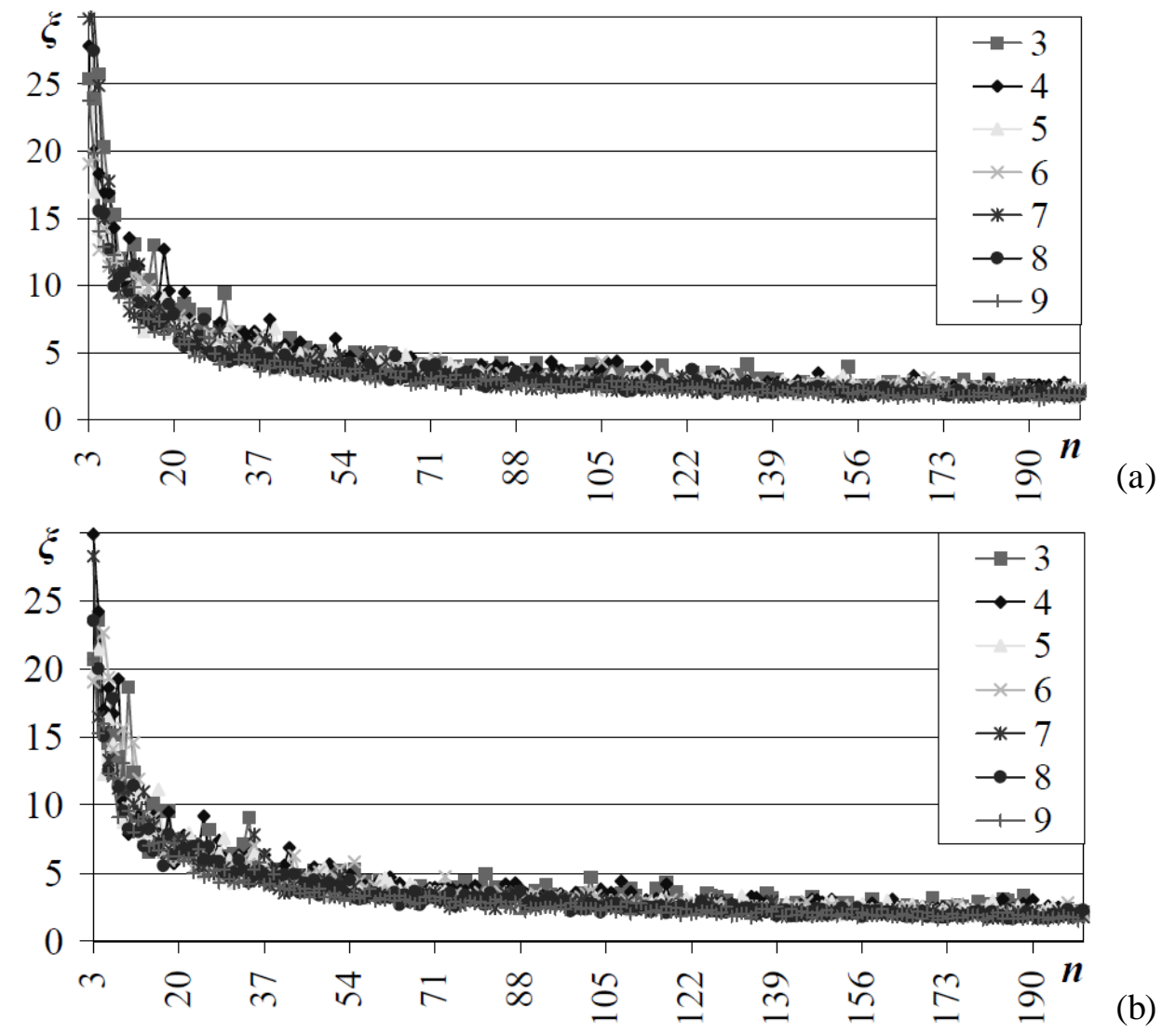

Fig.3. Dependences between difference of expert estimates $(\xi)$ for exponential (a) and half-normal (b) deviation distribution laws and the number of experts in a group $(n)$.

\section{Conclusions}

Expert competence can be neglected when the number of experts in a group exceeds few decades. Since in weakly-structured domains expert examinations are usually conducted by small expert groups, in such conditions taking expert competence into account is significant for getting a reliable result, and cannot be neglected.

\section{REFERENCES}

Liubchenko, V.V. (2005). A study of significance of taking competence factors into consideration while group decision making [E-resource] Proceedings of Odessa Polytechnic University, 1(23), Access mode: http://storage.library.opu.ua/online/periodic/opu_2005_1(23)/5/5_2.pdf. (in Russian)

Miller, G.A. (1956). The magical number seven, plus or minus two: Some limits on our capacity for processing information. The Psychological Review, 63(2): 81-97.

Orlov, A.I. (1996). The expert estimations. Factory laboratory, 62(1): 54-60. (in Russian)

Saaty, T.L. (1980). The Analytic Hierarchy Process. New York, NY: McGraw-Hill.

Saaty, T.L. (2008). Relative measurement and its generalization in decision making. Why pairwise comparisons are central in mathematics for the measurement of intangible factors. The Analytic Hierarchy/Network Process. Statistics and Operations Research, 102 (2): 251-318. 
Zagoruiko, N.G. (1988). The credibility of the information and its source in expert systems. Expert Systems and Pattern Recognition, 126: 3-23. (in Russian) 